\title{
Incidence rate and basic reproduction number of Coronavirus diseases (COVID-19): a systematic review and meta-analysis protocol
}

Hailay Abrha Gesesew ( $\nabla$ hailushepi@gmail.com )

Mekelle University College of Health Sciences

William Mude

Central Queensland University

Tafadzwa Nyanhanda

Central Queensland University

Lillian Mwanri

Flinders University Faculty of Medicine Nursing and Health Sciences

\section{Protocol}

Keywords: COVID-19, Coronavirus, incidence rate, reproduction number, incubation period, mortality rates, Ro, systematic review protocol

Posted Date: April 20th, 2020

DOl: https://doi.org/10.21203/rs.3.rs-22962/v1

License: (c) (i) This work is licensed under a Creative Commons Attribution 4.0 International License. Read Full License 


\section{Abstract}

Background : World Health Organization declared Coronavirus (COVID-19) as a newly emerging disease on 31 December 2019. In a space of two-months, there have been several studies reporting incidence rates, incubation periods, fatality rates, basic and effective reproduction numbers of the disease. However, the data have not been synthesized and pooled together to determine the global average.

Objective : The present study aims to systematically review available global evidence on incidence rates, and basic and effective reproduction numbers of COVID-19.

Methods : This is a protocol for a systematic review of available quantitative studies published in English. Studies which describe the incidence rates, and basic and effective reproduction numbers of COVID-19 will be included in the search. Initially, search term concepts will be constructed through a preliminary search on Google scholar. Then, we will perform a full systematic search using the identified concepts in the following electronic bibliographic databases: SCOPUS, PubMed, Web of Sciences and CINAHL. Preferred Reporting Items for Systematic Reviews and Meta-Analyses (PRISMA) will be used to schematically present the full search strategy. Standardised Joanna Briggs Institute (JBI) quality appraisal and selection tools, and data extraction tools will be used to recruit studies and extract data from the included studies, respectively. Thematic analysis will be used to analyse the non-quantifiable data, and meta-analysis will be used to pool the quantitative information.

\section{Introduction}

World Health Organization (WHO) [1] declared the 2019 novel coronavirus on 31 December 2019 as a new and emerging disease following a report of cluster of pneumonia cases in Wuhan, China[2]. The clinical spectrum of COVID-19 has varied on the degree of severity $[3,4]$. In mild cases, patients have presented with non-pneumonia or mild pneumonic symptoms such as fever, malaise and dry cough. However, $14 \%$ of severe cases, patients present have presented with dyspnea, respiratory frequency of is more than $30 / \mathrm{min}$ and blood oxygen saturation $\left(\mathrm{SpO}_{2}\right)$ of below 93\%[3]. Patients who were at a critical stage (occurring in $5 \%$ of cases) may experience septic shock, respiratory failure, and multiple organ failure[3]. COVID-19 is presumed to have originated from be animal-to-human transmission. Human-tohuman transmission now occurs occur through respiratory droplets via coughing and, sneezing or coming in contacts with contaminated surfaces[3].

The first COVID-19 related death was confirmed on 9 January 2020[2] in China. The first case outside China was confirmed in Thailand on 13 January 2020[1]. As of 28 March 2020, 3:30 PM (UTC+10:30 Adelaide time), there were 597,304 confirmed cases over 176 countries with the top ten hardest hit countries including the United States of America $(104,661)$, Italy $(86,498)$, China $(81,946)$, Spain $(65,719)$, Germany $(50,871)$, France $(33,414)$, Iran $(32,332)$, the United Kingdom $(14,751)$, Switzerland $(12,928)$ and South Korea $(9,478)[5]$. To date (28 March 2020), there have been over 27,364 deaths and the following countries had the highest number of death records[5]: Italy $(9,134)$, Spain $(5,138)$, China $(3,296)$, Iran 
$(2,378)$, France $(1,995)$, The United States of America $(1,296)$, United Kingdom (759), Netherland $(546)$, Germany (351) and Belgium (289). WHO reports that the global case fatality rate as of 23 March 2020 was $4.4 \%[6]$, ranging up to $7.2 \%$ in Italy[7].

The WHO declared COVID-19 as Public Health Emergency of International Concern (PHEIC) on 30 January 2019[8] and a as a pandemic on 11 March 2020[9]. In its short life, of less than two months, this pandemic has swiftly caused significant socio-economic, cultural and political crisis across the world[10]. A number of countries have declared a state of emergency which has led to many changes globally, including cancellation of international and domestic flights, curtailing of essential travel by closing international and state borders between and within countries, closing public spaces and events and changing the way businesses are operated such as universities changing teaching modalities and teaching virtually[10]. These abrupt changes have led to significant impacts on communities and individuals such unemployment, loss of social network and social capital[10].

Given the disease is now spread to 176 countries, evidence from available data provides a differing picture about the natural history and spectrum of the disease i.e. incubation period, infectivity, pathogenicity and virulence, and basic reproduction number. For example, the China Center for Disease Control and Prevention (CDC) and local CDCs, using data from the first cases in Wuhan, reported a 3-7 days of incubation time, 9.2 to 18 days of infectious period, and 2.2 basic reproduction number $\left(R_{0}\right)$ [11]. Furthermore, the incidence, prevalence and mortality rates vary from country to country. For example, while Europe is becoming the hotline,Africa is the least affected at this stage[8].

Obtaining exact data for these important variables is significant in mapping the number and speed of transmission. As the virus does not have a specific treatment, the only viable solution is halting the transmission through isolation or quarantine. Furthermore, having correct data is important to drive the implementation of new of mitigation strategies. For example, some countries have ordered a complete lockdown[12], others are allowing essential daily business and planning to adapt a 'new normal'[13], and some are still conducting routine activities normally. We therefore need to have a pooled data of these variables to build evidence that will inform effective and seamless contextual policies and practices.

The aim of the present study is to synthesize and (pool using meta-analysis) global data of incidence and basic reproduction number of COVID-19. We will also synthesize evidence on the incubation period, infection time and mortality rates of COVID-19.

\section{Methods}

\section{Population}

In the systematic review, we will include studies on incidence and basic reproduction number of COVID19 among any population group across the world. 


\section{Study design}

We will consider all quantitative studies of good quality published in English between 29 December 2019 and 31 March 2020.

\section{Search strategy}

We will carry out the following steps to perform the search strategy. First, we will perform a limited search through Google scholar to construct concepts and key terms for three pre-defined concepts namely: concept 1 (incidence, new cases, magnitude), concept 2 (basic reproduction number, $R_{0}$ ), and concept 3 (Coronavirus, COVID-19, SARS COV-2). Second, we will carry out a full search (Annex 1) using the identified keywords of the constructed concepts and key terms across the following electronic bibliographic databases: SCOPUS, PubMed, Web of Sciences and CINAHL. Concepts 1 and 2 will be connected using 'OR' and then connected with concept 3 with 'AND' to run the full search strategy in identified databases. Third, titles and abstracts will be screened from each database in order to select relevant document for a full-text critical appraisal. Fourth, bibliographies of relevant documents will be checked manually for additional studies. In addition, grey literature of unpublished studies will be explored from several resources such as Health department Data, World Health Organization (WHO), ProQuest Dissertations and Theses (PQDT), and other repositories for health data. The search strategy process will be schematically presented using the Preferred Reporting Items for Systematic Reviews and Meta-Analyses protocols (PRISMA-P) guidelines.

\section{Study selection and quality assessment}

The selected papers will be critically appraised for methodological quality independently by two reviewers (HAG and WM) using a standardised Joanna Briggs Institute (JBI) appraisal instruments[14] (Annex 2). The appraisal tool has nine questions to appraise descriptive and cohort studies, ten questions for experimental studies, and 11 questions for systematic review studies. Then, scores for methodological quality for each article will be calculated using the number of yes's $(Y)$ as the numerator, and the sum of unclear ('U'), no ('N's) and ' $Y$ ' as the denominator. Not applicable ('NA') will be excluded from the calculation. Any disagreements between the reviewers will be resolved by consensus or a third person, TN. The Agency for Healthcare Research and Quality (AHRQ) criteria[15] will help us to assess the risk of bias. The instrument evaluates selection bias, detection bias, performance bias, reporting bias and attrition bias [16]. The tool consists of the following values: high, moderate, low or unclear risk of bias [17]. A bias is detected as high ifthere is a substantial bias that invalidate the findings. This could be demonstrated by error at design, analysis or reporting stage. A bias is detected as moderate if there is a susceptible bias that potentially invalidate findings but enough evidence is not recorded. This could be demonstrated by if data is missed to assess the study's limitations. A bias is detected low if there is low bias but findings are valid. This could be demonstrated by low attrition or dropout rate, appropriate measurement/s of outcome, data analysis and reporting. A bias is detected unclear if it isdifficult to judge 
and studies are assumed to be reported poor. We will use the Grades of Recommendation, Assessment, Development and Evaluation (GRADE) system to grade the quality of evidence.

\section{Data extraction}

Data will be extracted using the standard JBI data extraction instrument (Annex 3). To extract relevant data from all articles included in the review, we will develop a table in word document consisting the following variables: Year of publication, Country (countries) of study setting, Study design, Population, Sample size, Outcome/s, and Summary of findings. We will contact primary authors in cases some data are missed or unclear.

\section{Outcomes}

Incidence and basic reproduction number of COVID-19 will be the primary outcomes in the review. Incidence refers to the number of new COVID-19 cases diagnosed within a particular period of time. The diagnosis of COVID-19 will be based on the laboratory and clinical criteria[3]. Incubation period, communicability period, and COVID-19 related mortality rates will be the secondary outcome of the review.

\section{Data management and analysis}

We will produce a narrative synthesis of outcomes in the final review. We will also summarize key findings from the included studies, and perform meta-analyses using RevMan-5 Software[18] to assess the association between the primary outcome and exposure. To do meta-analysis, (i) the research team will check clinical heterogeneity, and (ii) standard Chi-square and $\mathrm{I}^{2}$ tests $\left(\mathrm{I}^{2}<85 \%\right.$, $\mathrm{P}$ value $\left.<0.05\right)$ will be used to assess statistical heterogeneity[19]. We will use forest plots to present the pooled incidence and basic reproduction number graphically. Based on the level of statistical heterogeneity[19], random or fixed effect model will be used to produce the pooled estimates and their $95 \%$ confidence intervals $(\mathrm{CI})$. If no heterogeneity, we will use fixed effect model, and if moderate level of heterogeneity $\left(I^{2}<85 \%\right)$, we will use random model. At least two studies should assess the outcome of interest in order to pool to metaanalysis. Funnel plot will be used to assess the publication bias.

\section{Conclusions}

The systematic review will provide evidence of the magnitude and reproduction number of COVID-19. We will apply descriptive and inferential statistical analysis to summarise the quantitative data. We also use thematic analysis to synthesise the qualitative information of the findings. The review will provide a vital evidence for public health interventions to control and halt the transmission of COVID-19. 


\section{List Of Abbreviations}

COVID-19: coronavirus disease; PRISMA-P: Preferred Reporting Items for Systematic Reviews and MetaAnalyses Protocols; JBI- Joanna Briggs Institute; WHO: World Health Organization; CDC: Center for Disease Control and Prevention; $\mathrm{R}_{0}$ :basic reproduction number; AHRQ: Agency for Healthcare Research and Quality.

\section{Declarations}

Ethics approval and consent to participate: Ethics approval will not be required as there will be no primary data collection. We will disseminate the findings through peer-reviewed publications, conference presentations, and media releases.

Consent for Publication: Not applicable

Availability of data and material: All the necessary data are included in the manuscript.

Competing interests:The authors declare that they have no competing interest.

Funding:Not Applicable

Authors' contributions:HAG, WM, TN and LM involved in conceiving the idea and designing of the protocol. HAG wrote the original draft. HAG,WM, TN and LM critically review the protocol. All authors approved the final version of the protocol.

Acknowledgments:The authors are grateful to Flinders University and Central Queensland University for providing free access to articles.

Authors Information:HAG is an Associate Professor at the department of Epidemiology at Mekelle University, Ethiopia and Research fellow at the College of Medicine and Public Health at Flinders University, Australia. WM is a lecturer at the department of Public Health in Medical and Applied Sciences, Central Queensland University, Australia. TN is a senior lecturer at the department of Public Health in Medical and Applied Sciences, Central Queensland University, Australia. LM is an associate professor and course coordinator at the College of Medicine and Public Health at Flinders University, Australia.

\section{References}

1.Novel Coronavirus-Thailand (ex-China) /https://www.who.int/csr/don/14-january-2020-novelcoronavirus-thailand-ex-china/en/]

2.Boldog P, Tekeli T, Vizi Z, Dénes A, Bartha FA, Röst G: Risk Assessment of Novel Coronavirus COVID-19 Outbreaks Outside China. Journal of clinical medicine 2020, 9(2). 
3.Cascella M, Rajnik M, Cuomo A, Dulebohn SC, Di Napoli R: Features, Evaluation and Treatment Coronavirus (COVID-19). In: StatPearls [Internet]. edn.: StatPearls Publishing; 2020.

4.Wu Z, McGoogan JM: Characteristics of and Important Lessons From the Coronavirus Disease 2019 (COVID-19) Outbreak in China: Summary of a Report of 72314 Cases From the Chinese Center for Disease Control and Prevention. Jama 2020.

5. Coronavirus COVID-19 Global Cases by the Center for Systems Science and Engineering [https://coronavirus.jhu.edu/map.html]

6. The global coronavirus fatality rate has doubled in just 2 months, according to WHO data https://www.businessinsider.com.au/coronavirus-covid-19-chart-graph-global-fatality-rate-change-time$2020-3 ? r=$ US\&IR $=\mathrm{T}]$

7.Livingston E, Bucher K: Coronavirus Disease 2019 (COVID-19) in Italy. Jama 2020.

8. Statement on the second meeting of the International Health Regulations (2005) Emergency Committee regarding the outbreak of novel coronavirus (2019-nCoV) /https://www.who.int/news-room/detail/3001-2020-statement-on-the-second-meeting-of-the-international-health-regulations-(2005)-emergencycommittee-regarding-the-outbreak-of-novel-coronavirus-(2019-ncov)]

9. World Health Organization Declares COVID-19 a 'Pandemic.' /https://time.com/5791661/whocoronavirus-pandemic-declaration/]

10.COVID-19 crisis: Politcial and economic aftershocks /https://www.fpri.org/article/2020/03/covid-19crisis-political-and-economic-aftershocks/]

11.Li Q, Guan X, Wu P, Wang X, Zhou L, Tong Y, Ren R, Leung KSM, Lau EHY, Wong JY et al: Early Transmission Dynamics in Wuhan, China, of Novel Coronavirus-Infected Pneumonia. The New England journal of medicine 2020.

12.Italy Locks Down Much of the Country's North Over the Coronavirus [https://www.nytimes.com/2020/03/07/world/europe/coronavirus-italy.html]

13.PM orders total shutdown of all non-essential services from today 23 March 2020 [https://www.skynews.com.au/details/_6143840378001]

14.JBI: Joanna Briggs Institute. Joanna Briggs Institute reviewers' manual. In. Adelaide, SA; 2014.

15.Viswanathan M, Ansari MT, Berkman ND, Chang S, Hartling L, McPheeters M, Santaguida PL, Shamliyan T, Singh K, Tsertsvadze A et al: AHRQ Methods for Effective Health Care: Assessing the Risk of Bias of Individual Studies in Systematic Reviews of Health Care Interventions. In: Methods Guide for Effectiveness and Comparative Effectiveness Reviews. edn. Rockville (MD): Agency for Healthcare Research and Quality (US); 2008: 14. 
16.Viswanathan M: Assessing the Risk of Bias of Individual Studies in Systematic Reviews of Health Care Interventions. In., edn.: AHRQ (US Agency for Healthcare Research and Quality); 2012.

17.0wens DK, Lohr KN, Atkins D, Treadwell JR, Reston JT, Bass EB, Chang S, Helfand M: AHRQ series paper 5: grading the strength of a body of evidence when comparing medical interventions-agency for healthcare research and quality and the effective health-care program. J Clin Epidemiol 2010, 63(5):513523.

18.Cochrane: Review Manager (RevMan) [Computer program] In., 5.3 edn. Copenhagen: The Nordic Cochrane Centre, The Cochrane Collaboration; 2014.

19.Presenting and interpreting meta-analyses: Heterogenity

[http://www.nottingham.ac.uk/nmp/sonet/rlos/ebp/meta-analysis2/4.html]

\section{Supplementary Files}

This is a list of supplementary files associated with this preprint. Click to download.

- Supplimentaryfiles.docx

- PRISMAPchecklist.docx 